\title{
Schönig, Barbara; Kadi, Justin; Schipper, Sebastian (Hrsg.) (2017): Wohnraum für alle?! Perspektiven auf Planung, Politik und Architektur
}

\author{
Bielefeld: Transcipt. 356 Seiten
}

\section{Karin Wiest ${ }^{1}(\mathbb{D}$}

Online publiziert: 18. Januar 2018

๑) Springer-Verlag GmbH Deutschland, ein Teil von Springer Nature 2018

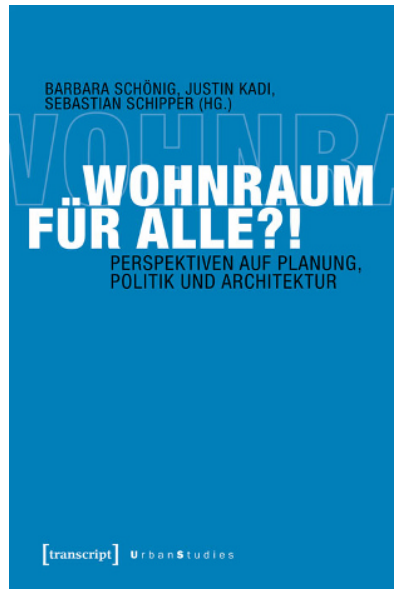

Ausgangspunkt des Sammelbandes „Wohnraum für alle?! Perspektiven auf Planung, Politik und Architektur" sind die hohen Miet- und Immobilienpreise, die in deutschen und europäischen Städten insbesondere seit der globalen Finanzkrise im Jahr 2008 sprunghaft angestiegen sind. Die Frage, wie eine bezahlbare Wohnraumversorgung unter diesen Bedingungen ausgestaltet und langfristig sichergestellt werden kann, gilt derzeit für die großstädtische Kommunalpolitik als eine der grundlegendsten Herausforderungen. Die Autoren des Bandes aus den Bereichen Stadtplanung, Soziologie, Geographie, Architektur und aus der Politik werfen aus unterschiedlichen Perspektiven Licht auf dieses drängende Problem und setzen sich marktkritisch mit den zugrunde liegenden politisch-ökonomischen Wirkmechanismen der neuen Wohnungsnot auseinander. Unterschiedliche Strategien und Experimentierfelder, wie eine nicht

Dr. Karin Wiest

k_wiest@ifl-leipzig.de

1 Leibniz-Institut für Länderkunde,

Schongauerstraße 9, 04329 Leipzig, Deutschland warenförmige Wohnungsversorgung ausgestaltet werden könnte, stehen dabei zur Diskussion. Präsentiert werden wissenschaftliche Reflexionen und konkrete Erfahrungen aus Planungspraxis, Politik, sozialen Bewegungen sowie gestalterische Lösungsansätze.

Der Sammelband steht deutlich in der Tradition einer kritischen Stadtforschung, die Fragen zur gesellschaftlichen Produktion von urbanen Ungleichheiten, Gentrifizierung und sozialräumlichen Exklusionen in den Mittelpunkt ihrer Analysen stellt und auf eine Veränderung von politischen und sozialen Entwicklungen abzielt (z.B. Brenner/ Marcuse/Mayer 2011; Belina/Heeg/Pütz et al. 2013; Belina/ Naumann/Strüver 2014; Gintrac 2016). Dementsprechend stellt Barbara Schönig die grundlegende Verknüpfung von Fragen sozialer Gerechtigkeit und Fragen der Wohnversorgung in das Zentrum ihrer einleitenden Thesen: Die neue Brisanz der Wohnungsfrage wird in historischer Perspektive als Resultat einer zunehmenden Durchsetzung neoliberaler Politiken auf unterschiedlichen - insbesondere lokalen und nationalen - Ebenen sowie auf die Funktionsmechanismen globaler Finanz- und Wirtschaftsströme zurückgeführt. Darauf aufbauend stellt das einleitende Kapitel ,die Rückkehr der Wohnungsfrage und ihre Ursachen“ - so der Titel - ins Zentrum. Es versammelt fünf Beiträge, die sich dem Thema vor allem aus einer gesellschaftstheoretischen Perspektive annähern und die Zusammenhänge zwischen Finanz- und Wohnungsmärkten auf globalen, nationalen und lokalen Ebenen beleuchten.

Bernd Belina bettet seine Kritik an der Kapitalisierung des Wohnungsmarktes ein in eine schematische Darstellung unterschiedlicher Kapitalkreisläufe und ihrer supranationalen Einbindung. Susanne Heeg ergänzt diese Analyse um eine weitere in weltwirtschaftliche Zusammenhänge eingebundene Betrachtung, die die Vermarktlichung und Ökonomisierung von Wohnungen zusammen mit dem Rückbau des Wohlfahrtsstaates als zentrale preistreibende 
Faktoren auf den städtischen Wohnungsmärkten beschreibt. Ergänzend beleuchten zwei Autorinnen den gemeinnützigen Wohnsektor aus europäischer Perspektive: sie thematisieren die Bedeutung nationaler Unterschiede des sozialen Wohnungsbaus (Kathleen Scanlon) und den Einfluss des europäischen Wettbewerbsrechts auf das Sozialwohnungswesen (Darinka Czischke). Susanne Frank nimmt schließlich mit den neuen Mittelschichten die Nachfragerseite von Gentrifizierungsprozessen in den Blick. Spannend ist ihre These, dass die zunehmende Kritik an der Gentrifizierung - als mächtigem Symbol für die neoliberale Stadt - ähnlich wie im Fall der Suburbanisierung, zu einer Veränderung von gesellschaftlichen Wohnidealen führen könnte. Inwieweit eine Abkehr der trendsetzenden Mittelschichten vom Wunsch, in attraktiven innerstädtischen Lagen zu leben, sich derzeit schon abzeichnet, wäre allerdings noch zu klären. Die Frage, inwieweit die neuen sozialen Bewegungen in der Lage sind, den dominanten Kurs der Deregulierung, Privatisierung und Kapitalisierung des Städtischen zu beeinflussen, sind das zentrale Thema eines Städtevergleichs im zweiten Kapitel des Sammelbandes (Anne Vogelpohl, Lisa Vollmer, Elodie Vittu und Norma Brecht). Der Grad der Etablierung, Vernetzung und Sichtbarkeit der urbanen Protestbewegungen von unten kann in diesem Sinn durchaus als ein Erfolg interpretiert werden. Gleichwohl stellen privatwirtschaftliche Akteure, regierende Parteien und/oder hegemoniale Diskurse überstarke Gegenkräfte dar.

Im dritten Kapitel diskutieren Andrej Holm, Bernd Hunger und Jan Kuhnert in ihren jeweiligen Beiträgen die Notwendigkeit der Etablierung einer ,neuen` Gemeinnützigkeit, um - nach Aufhebung des Wohnungsgemeinnützigkeitsrechts zum 31. Dezember 1989 - die Herausforderungen der Wohnungsfrage in Städten sozial gerecht bewältigen zu können. Die gesellschaftliche Dringlichkeit, historische Prinzipien und Erwartungen an eine neue Gemeinnützigkeit werden differenziert, nachvollziehbar und ausführlich dargelegt. Relativ offen bleibt die Frage nach den Wegen, wie dieser befürwortete Ausbau politisch realisiert werden könnte, und nach den damit verbundenen Grenzen und Hindernissen.

Die Wirksamkeit von Instrumenten und konkreten Strategien der kommunalen Wohnungspolitik ist Gegenstand des vierten Kapitels (,Kommunale Strategien“). Ausgangspunkt bilden besonders jüngste Tendenzen eines schrittweisen Umdenkens in stadtentwicklungspolitischen Diskursen, die mit einer Renaissance wohnungspolitischer Regulierungsstrategien wie der Mietpreisbremse, Milieuschutzsatzungen oder der Ausweitung der öffentlichen Wohnungsbauförderung einhergehen. Vor diesem Hintergrund gehen Joscha Metzger und Sebastian Schipper der Frage nach, welche Gegenbewegungen sich zu den jahrzehntelangen Neoliberalisierungstendenzen in den Städten Hamburg und Frankfurt am Main beobachten lassen und inwieweit dies- bezüglich ein wohnungspolitischer Paradigmenwechsel beobachtbar ist. Das Wiener Modell, das vielfach als ein kommunales Vorzeigebeispiel für eine sozial gerechte Wohnungsversorgung gilt, wird im Beitrag von Christoph Reinprecht mit gegenwärtigen Herausforderungen in den $\mathrm{Zu}$ gangsmöglichkeiten zu bezahlbarem Wohnraum konfrontiert, und damit zum Teil entzaubert. Das im Jahr 2014 in Berlin eingeführte Modell der kooperativen Baulandentwicklung (Grit Schade) repräsentiert eine weitere Maßnahme, um über Kooperationsvereinbarungen zwischen Investoren und kommunaler Planungshoheit einen Beitrag für die soziale Wohnraumversorgung zu leisten. In Ergänzung dazu zeigt Katrin Lompscher den Weg zur Einführung des Berliner Wohnraumversorgungsgesetzes im Jahr 2016 in seinem bundes- und lokalpolitischen Kontext auf.

Bezugnehmend auf die dargestellten urbanen Kämpfe um das bezahlbare Wohnen (Kapitel 2) werden unter dem Titel „Alternativen jenseits von Staat und Markt“" in Kapitel 5 Modelle der Wohnraumversorgung im Feld kooperativer und demokratischer Selbstverwaltung behandelt: Genossenschaftliche Wohnprojekte (wie etwa das Mietshäuser Syndikat) (Ivo Balmer und Tobias Bernet) sowie Community Land Trusts in den USA als nicht gewinnorientierte, treuhänderisch verwaltende Gesellschaften (Sabine Horlitz). Gestalterische und architektonische Möglichkeiten, um die Kosten für den Wohnungsbau gering zu halten, runden den Sammelband im letzten Kapitel ab. Hier analysieren Jutta Albus und Hans Drexler die Potenziale vorgefertigter Konstruktionssysteme an Beispielen aus der Praxis in Spanien, Österreich und Deutschland. Einen Blick auf die Geschichte der deutschen Wohnungspolitik werfen Caroline Kauert und Max Welch Guerra, indem sie die Rolle des Bauhauses für die Lösung der Wohnungsfrage beleuchten und in den gesellschaftspolitischen Kontext des beginnenden 20. Jahrhunderts einbetten.

Fazit: Das Buch richtet sein Plädoyer für eine nicht profitorientierte Organisation der Wohnraumversorgung an städtische Akteure, Planer und Politiker - nicht zuletzt, um im Sinne einer engagierten Forschung eine Veränderung urbaner Verhältnisse zu bewirken. Die Wiederbelebung des sozialen Wohnungsbaus, staatliche und kommunale Regulierungsmaßnahmen, genossenschaftliche Modelle und neue bürgerschaftliche Bewegungen bilden dabei die zentralen Hebel, um eine Wohnraumversorgung zu organisieren, die - zumindest in Teilbereichen - den Mechanismen des Marktes entzogen ist. Eine Übersicht, die die jeweiligen Potenziale der dargestellten Strategien, die Ebenen ihrer Anwendbarkeit, ihre Reichweite sowie die Grenzen ihrer Umsetzbarkeit in Form eines zusammenfassenden Handlungskonzeptes aufzeigt, hätte diesem informativen, interdisziplinären Reader noch mehr Gewicht verleihen können. Die Umsetzung des eingangs formulierten Anliegens, auf der Grundlage einer Zusammenführung der 
fragmentierten wissenschaftlichen Diskurse tragfähige, interdisziplinäre Lösungsansätze abzuleiten, steht also noch aus.

\section{Literatur}

Belina, B.; Heeg, S.; Pütz, R.; Vogelpohl, A. (Hrsg.) (2013): Neuordnungen des Städtischen im neoliberalen Zeitalter - Zur Einleitung. In: Geographische Zeitschrift 101,3+4, 125-131.
Belina, B.; Naumann, M.; Strüver, A. (Hrsg.) (2014): Handbuch kritische Stadtgeographie. Münster.

Brenner, N.; Marcuse, P.; Mayer, M. (Hrsg.) (2011): Cities for People, not for Profit: Critical Urban Theory and the Right to the City. New York/London.

Gintrac, C. (2016): Kritische Stadtgeographie - ein Archipel epistemischer Gemeinschaften. In: Sublurban 4, 2/3, 59-82. 